\title{
Ketonemia and Seizures: Metabolic and Anticonvulsant Effects of Two Ketogenic Diets in Childhood Epilepsy
}

\author{
PETER R. HUTTENLOCHER (22) \\ Department of Pediatrics, University of Chicago, Chicago, Illinois, USA
}

\section{Extract}

Metabolic effects of a new ketogenic regimen in which ketonemia is induced by feeding of medium chain triglycerides (MCT) are described, and comparisons are made with effects of the standard high fat ketogenic diet. Eighteen children maintained on the MCT diet for 3 months to 4 years failed to show elevations of serum cholesterol and had only a slight rise in serum total fatty acids, in contrast to the marked hyperlipidemia observed in children on the standard high fat diet. Long term use of the MCT diet did not affect $\mathrm{pH}$ of venous blood. Blood glucose fell below $50 \mathrm{mg} / 100 \mathrm{ml}$ in one-third of the children, the lowest levels being reached 2-3 weeks after the start of the diet. Plasma D(-)- $\beta$-hydroxybutyrate (BHB) and acetoacetate rose gradually after institution of diet therapy, maximum levels being reached after about 1 month. Higher levels of BHB and acetoacetate were achieved in children under the age of 10 years $(\mathrm{BHB}=4.3 \mathrm{mM} \pm 0.6 \mathrm{SEM}$, acetoacetate $=1.8$ $\mathrm{mM} \pm 0.3 \mathrm{SEM})$ than in $10-18$ year olds $(B H B=1.6 \mathrm{mM} \pm 0.2$ SEM, acetoacetate $=0.57 \mathrm{mM} \pm 0.08$ SEM). Plasma BHB and acetoacetate levels in children maintained on a 3:1 high fat diet were similar to those in children on a $60 \%$ MCT diet. Plasma levels of BHB showed a significant correlation with anticonvulsant effect $(P<0.02)$. Both the ketonemia and the anticonvulsant action were reversed rapidly by intravenous infusion of glucose.

\section{Speculation}

The anticonvulsant effect of the standard ketogenic diet and of the MCT diet are dependent upon maintenance of blood levels of BHB above $2 \mathrm{mM}$ and of acetoacetate above $0.6 \mathrm{mM}$. The data suggest that one or both of these compounds either have direct anticonvulsant effects or produce rapidly reversible changes in cerebral metabolism, which in turn affect cerebral excitability.

The anticonvulsant effect of ketogenic regimens, in particular of the high fat, low carbohydrate ketogenic diet, has been known for over 50 years (15). However, observations regarding the mechanism of their anticonvulsant action have been limited. Early clinical investigations suggested that the anticonvulsant effect may be directly related to ketonemia $(8,13)$. Subsequently, several other possible mechanisms have been proposed. It has been suggested that the effect may be secondary to $\mathrm{pH}$ change. However, experimental evidence concerning an anticonvulsant effect of acidosis is scantly, and available data indicate that the means by which $\mathrm{pH}$ is lowered rather than acidosis per se may be of importance (4). Furthermore, although metabolic acidosis has been documented soon after institution of the high fat ketogenic diet $(3,9)$, there is no evidence that blood $\mathrm{pH}$ is decreased in patients on long term ketogenic regimens (3). Other suggested mechanisms include a possible anticonvulsant effect of hyperlipidemia (2); an effect of carbohydrate restriction on cerebral metabolism with change from dependence on glucose as a substrate for energy metabolism to direct utilization of $\beta$-hydroxybutyrate $(1,6,14)$; and an effect of the ketogenic diet on sodium and potassium balance (9). Definite evidence for any of these is presently lacking.

We have recently used a new ketogenic regimen in which ketonemia is induced by feeding of MCT (6). The MCT diet has anticonvulsant effects which resemble those of the standard high fat ketogenic diet $(6,7)$. The availability of two ketogenic diets which differ in some metabolic effects has made it possible to further define those factors which appear to be of importance for anticonvulsant action. The present study reports some metabolic changes induced by the MCT diet and compares them with the high fat 3:1 ketogenic diet. In addition, data have been obtained on the effect of glucose infusion in children maintained on a ketogenic diet, and on correlations between plasma ketone levels and anticonvulsant effects. The findings support a direct anticonvulsant action of ketone bodies, either BHB, acetoacetate, or both.

\section{CASE MATERIALS AND METHODS}

Metabolic studies were carried out in 18 children maintained on the MCT diet and in 4 maintained on a standard 3:1 ketogenic diet. The age range of patients was 18 months to 18 years. All had had extensive trials on anticonvulsant drugs in various combinations and were thought to be refractory to therapy before the start of the ketogenic diet. Predominant seizure types were as follows: minor motor (akinetic or myoclonic), 11 patients; mixed minor motor and grand mal, 4; focal or multifocal motor seizures, 3; psychomotor seizures, 3 ; petit mal, 1 . Recognizable diseases underlying the seizure disorder were present in two: one each of Sturge-Weber disease and Fahr's disease (idiopathic calcification of cerebral cortex).

Before the start of the ketogenic diet the children were fasted until the urine became strongly positive for ketones (usually for about $48 \mathrm{hr}$ ). The composition of the MCT diet and of the standard 3:1 ketogenic diet used in this study is given in Table 1. MCT was incorporated into foods and given as an MCT-skimmed milk drink as outlined in a previous report (6). The MCT-skimmed milk mixture was served well chilled, in small sips throughout each meal. Initially, total caloric intake was kept at a low maintenance level (about $75 \mathrm{cal} / \mathrm{kg} / 24 \mathrm{hr}$ ) and caloric intake was gradually increased if the child was hungry or if he lost weight. In seven children, the diet was maintained for over I year, with a maximum of four years. The majority of the remainder had therapeutic trials lasting 3-6 months.

$D(-)-\beta$-hydroxybutyrate and acetoacetate levels in plasma and cerebrospinal fluid (CSF) were measured by the method of Williamson et al. (16), which utilizes the interconversion of these two substances by the enzyme $\mathrm{D}(-)-\beta$-hydroxybutyrate dehydrogenase. Plasma samples were deproteinized by addition of equal volumes of $30 \%$ ice-cold perchloric acid. For determination of $\mathrm{BHB}$, 
Table 1. Approximate proportions of principal dietary constituents in medium chain triglyceride (MCT) and 3:1 ketogenic diets

\begin{tabular}{lcc}
\hline & $\begin{array}{c}\text { MCT diet } \\
\text { (\% of total cal) }\end{array}$ & $\begin{array}{c}\text { 3:1 ketogenic diet } \\
\text { (\% of total cal) }\end{array}$ \\
\hline Carbohydrate & 18 & 6 \\
Protein & 10 & 7 \\
Dietary fat & 12 & 87 \\
MCT & 60 & 0 \\
\hline
\end{tabular}

aliquots of supernatant were added to a mixture of Tris buffer, $0.5 \mathrm{ml} 0.1 \mathrm{M}$ solution, $\mathrm{pH} 8.5 ; \mathrm{DPN}^{+}(18), 1 \mu \mathrm{mol}$; and hydrazine hydrate, $1 \mathrm{ml} 1 \mathrm{mM}$ solution, $\mathrm{pH}$ 8.5. The total volume was brought to $3 \mathrm{ml}$ with distilled water, and the extinction of the reaction mixture was read at $340 \mathrm{~nm}$. Then $0.025 \mathrm{ml} \mathrm{D}(-)-\beta$-hydroxybutyrate dehydrogenase suspension (19) was added to the mixture and the decrease in extinction was read against a control sample without added enzyme at 5-min intervals until stable readings were obtained (about $60 \mathrm{~min}$ ). Readings were compared with those obtained with known concentrations of BHB. The material used as standard was the sodium salt of DL- $\beta$-hydroxybutyric acid (18), which contains $50 \%$ sodium $\mathrm{D}(-)-\beta$-hydroxybutyrate.

For determinations of acetoacetate the reaction mixture consisted of $0.2-1-\mathrm{ml}$ aliquots of deproteinized serum, $1 \mathrm{ml} 0.1 \mathrm{M}$ potassium phosphate buffer, $\mathrm{pH} 7.0$, and DPNH (18), $0.1 \mathrm{ml} 5$ $\mathrm{mM}$ solution brought to a total of $3 \mathrm{ml}$ with distilled water. The extinction was read at $340 \mathrm{~nm}$. Then $0.025 \mathrm{ml}$ of BHB dehydrogenase suspension was added and the extinction was read at 5-min intervals until no further increase occurred, usually about $30 \mathrm{~min}$. The material used as standard was lithium acetoacetate (18).

Blood glucose was measured by the glucose oxidase method (5). Plasma cholesterol was determined by a direct method (17). Total plasma fatty acids were estimated by a modification of the hydroxamic acid method for determination of long chain fatty acid esters (12). The normal range by this method for plasma obtained in the fasting state is 7-14 mEq/liter. For correlation of $\mathrm{BHB}$ blood levels with anticonvulsant effects a simple scale of effect on seizure control was used: no effect, less than $50 \%$ decrease in seizure frequency compared with prediet level: fair control, 50-90\% decrease in number of observed seizures; good control, more than $90 \%$ decrease in number of observed seizures; excellent control complete daytime seizure control, with persistence of occasional nocturnal seizures in some patients.

\section{RESULTS}

\section{METABOLIC EFFECTS OF MCT DIET}

Effects on Plasma Lipids. Plasma cholesterol in 18 children on the MCT diet did not vary significantly from control values. Before the start of the diet mean cholesterol was $175 \mathrm{mg} / 100 \mathrm{ml}$ ( $\perp 12$ SEM). Mean plasma cholesterol in children maintained on the MCT diet for 1 week to over 1 year was $185 \mathrm{mg} / 100 \mathrm{ml}( \pm 7$ SEM, $P>0.1$ ). Total fatty acids (including triglycerides) in plasma rose slightly compared with prediet levels, from 10.8 to 14 $\mathrm{mEq} /$ liter $(P=0.025)$.

Determination of plasma lipids in four children maintained on the 3:1 ketogenic diet showed elevations of plasma cholesterol, with a mean of $266 \mathrm{mg} / 100 \mathrm{ml}$ and of plasma total fatty acids (mean $=18 \mathrm{mEq} /$ liter). These results are in agreement with more extensive prior data on hyperlipidemia in children maintained on a standard ketogenic diet (2).

Effects on Acid-Base Balance. Chronic use of the MCT diet was not associated with significant changes in blood $\mathrm{pH}$. Mean venous $\mathrm{pH}$ before the start of the diet was $7.36 \pm 0.01 \mathrm{SEM}$, whereas mean $\mathrm{pH}$ in children maintained on the diet for periods of up to 1 year was $7.37 \pm 0.01 \operatorname{SEM}(N=46)$. A few determinations of venous $\mathrm{pH}$ in children maintained for several months on the $3: 1$ ketogenic diet also gave normal values.
Effects on Blood Glucose. A mild depression in glucose levels occurred frequently, especially within the first month after the start of the MCT diet. Fasting blood glucose values before the start of the diet and at 1 week after the start were above $50 \mathrm{mg} / 100 \mathrm{ml}$ in all children. Two weeks after the start of the diet, values between 40 and $50 \mathrm{mg} / 100 \mathrm{ml}$ occurred in $33 \%$ of the children. Twentythree percent of glucose values obtained in children on dietary therapy for over 1 month fell in the $40-50 \mathrm{mg} / 100 \mathrm{ml}$ range. No values below $35 \mathrm{mg} / 100 \mathrm{ml}$ were observed, and none of the children were thought to have clinical signs of hypoglycemia.

$B H B$ and Acetoacetate Levels in Plasma. Plasma levels of BHB and acetoacetate were measured in 18 children maintained on the MCT diet and in 4 maintained on a 3:1 ketogenic diet regimen. Plasma levels of BHB were low while the children were on a normal diet (mean $=0.22 \mathrm{mM}$, range $0.06-0.4 \mathrm{mM}, n=8)$. Levels rose rapidly during fasting. Plasma levels at the end of fasting, before the start of the ketogenic diet, are listed in Table 2, as are levels achieved during dietary therapy. In children under age 10 years, the elevation in BHB obtained with the $60 \%$ MCT diet was comparable with that seen on the $3: 1$ ketogenic diet. With both diets, BHB levels increased during the first month of dietary therapy and then remained fairly stable as long as the diet was maintained without break. Four children above age 10 years were found to achieve significantly lower blood levels of BHB than did younger children on the same proportionate intake of MCT, carbohydrate, protein, and dietary fat. Difficulties inherent in the maintenance of the $3: 1$ ketogenic diet in older children or adolescents precluded observations on the effects of this diet in patients over 10 years of age.

Plasma levels of acetoacetate paralleled those of BHB and fell between $30 \%$ and $40 \%$ of BHB values (Table 3). Acetoacetate levels in plasma increased during the first month of diet therapy in children under age 10 years. This effect was absent in the above 10-year-old group, in which mean acetoacetate levels were only about a third as high as in the younger children.

BHB and acetoacetate levels in CSF were measured in two patients 4 days after start of the MCT diet. Mean CSF level of

Table 2. Plasma $D(-)-\beta$-hydroxybutyrate levels in millimolar concentrations during fasting, medium chain triglyceride (MCT), and 3:1 ketogenic diet therapy

\begin{tabular}{|c|c|c|c|c|c|c|}
\hline & \multicolumn{3}{|c|}{ Age $2-9$ years } & \multicolumn{3}{|c|}{ Age $10-18$ years } \\
\hline & Mean & SEM & No. & Mean & SEM & No. \\
\hline Fasting & 5.6 & 0.8 & 14 & 4.6 & 2.3 & 4 \\
\hline MCT diet $<1$ month & 2.9 & 0.4 & 25 & 1.2 & 0.2 & 6 \\
\hline MCT diet $>1$ month & 4.3 & 0.6 & 31 & 1.6 & 0.2 & 21 \\
\hline $\begin{array}{l}3: 1 \text { ketogenic diet } \\
\quad<1 \text { month }\end{array}$ & 2.4 & 0.9 & 6 & & & \\
\hline $\begin{array}{l}\text { 3:1 ketogenic diet } \\
>1 \text { month }\end{array}$ & 4.8 & 1.0 & 11 & & & \\
\hline
\end{tabular}

Table 3. Plasma acetoacetate in millimolar concentrations during fasting, medium chain triglyceride (MCT), and 3:1 ketogenic diet therapy

\begin{tabular}{|c|c|c|c|c|c|c|}
\hline & \multicolumn{3}{|c|}{ Age 2-9 years } & \multicolumn{3}{|c|}{ Age $10-18$ years } \\
\hline & Mean & SEM & No. & Mean & SEM & No. \\
\hline Fasting & 1.66 & 0.26 & 8 & 1.47 & & 3 \\
\hline MCT diet $<1$ month & 1.15 & 0.15 & 16 & 0.58 & 0.11 & 4 \\
\hline MCT diet $>1$ month & 1.81 & 0.30 & 15 & 0.57 & 0.08 & 16 \\
\hline $\begin{array}{l}3: 1 \text { ketogenic diet } \\
\quad<1 \text { month }\end{array}$ & 0.95 & 0.36 & 4 & & & \\
\hline $\begin{array}{c}\text { 3:1 ketogenic diet } \\
>1 \text { month }\end{array}$ & 1.84 & 0.29 & 7 & & & \\
\hline
\end{tabular}


BHB was $0.33 \mathrm{mM}$ or $12 \%$ of simultaneous plasma BHB; mean CSF level of acetoacetate was $0.30 \mathrm{mM}$ or $20 \%$ of simultaneous plasma acetoacetate. These results suggest that the CSF to plasma ratio of acetoacetate may be higher than that of $\mathrm{BHB}$, but further data are clearly needed to validate this point.

\section{RELATIONSHIPS BETWEEN KETONEMIA AND SEIZURE CONTROL}

Long Term Anticonvulsant Effects. On the same diet, different patients achieved different degrees of ketonemia. Both age and individual susceptibility to ketosis independent of age appeared to be factors. Mean BHB levels achieved during ketogenic diet therapy showed a relationship to seizure control (Fig. 1). A significantly greater proportion of children with mean BHB blood levels above $2 \mathrm{mM}$ achieved good to excellent seizure control than did children with mean blood level less than $2 \mathrm{mM}$ (chi-square $=5.8$, $P<0.02)$.

Effects of Glucose Infusion on Seizure Control. The effect of rapid reduction in plasma $\mathrm{BHB}$ and acetoacetate on the electroencephalogram and on clinical seizure activity was determined in three children. All three had been on the MCT diet for several months, two with good to excellent seizure control and one with fair control. In all three instances, a prior decision had been made to discontinue the MCT diet because the children or their parents were no longer able to tolerate the restrictions imposed by diet therapy. Base-line electroencephalographic tracings and blood studies were obtained. A $20 \%$ glucose solution was then infused intravenously at the rate of $20 \mathrm{~g} / \mathrm{hr}$ for $2 \mathrm{hr}$. Electroencephalogram tracings, plasma $\mathrm{BHB}$, acetoacetate, glucose, $\mathrm{pH}$, and $\mathrm{pCO}_{2}$ were measured repeatedly throughout the infusion.

Glucose infusion resulted in a rapid fall in plasma BHB and acetoacetate (Fig. 2). In two patients this was accompanied by deterioration of the EEG with appearance of spike and spike-wave discharges and increase in background sharp and slow wave activity (Fig. 3). Clinical seizures followed infusion of glucose in one of these two children, with onset of myoclonic jerks $60 \mathrm{~min}$ after the start of infusion (Fig. 3). The other child had a brief grand mal seizure $3 \mathrm{hr}$ after the start of infusion, but its relation to lowering of plasma ketones was unclear since this child had fairly frequent attacks while on diet therapy. The third child, in whom the background EEG remained unchanged, had a single myoclonic seizure $45 \mathrm{~min}$ after the start of the infusion, when his plasma BHB had fallen from 4.05 to $1.43 \mathrm{mM}$ (Fig. 2). Previously this child had been free of daytime seizures for 2 months.

\section{DISCUSSION}

Metabolic data have been collected in a group of 22 children maintained on ketogenic diets. The results lead to several conclusions regarding the mechanism of anticonvulsant action of diet therapy.

1. The effect does not appear to be secondary to hyperlipidemia, a mechanism previously suggested by Dekaban (2). This now appears unlikely, since the MCT and the 3:1 ketogenic diets, although approximately equal in anticonvulsant effect $(6,7)$, have very different effects on plasma lipid levels. Cholesterol, which is elevated in children on the 3:1 diet, remains normal on the MCT diet, and fatty acid levels show less increase on the MCT regimen.

2. Chronic extracellular acidosis does not appear to be a factor in the anticonvulsant effect. Blood $\mathrm{pH}$ in children maintained on long term diet therapy has been found to be normal, as these children apparently have adequate respiratory compensation for the rise in acid anions.

3. Mild hypoglycemia is an inconstant finding in children on the MCT diet and does not correlate with anticonvulsant effect. Extensive data on blood glucose levels in children maintained on the standard ketogenic diet are not available. De Vivo et al. (3) recently reported marked hypoglycemia in one child after start of the $3: 1$ ketogenic diet, and mention the occurrence of mild transient hypoglycemia as a common finding in children maintained on the high fat diet.

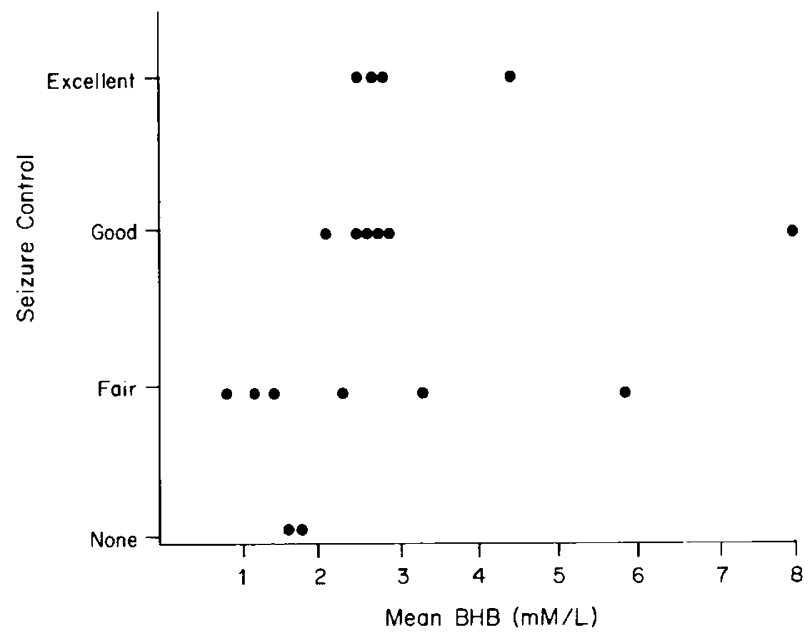

Fig. 1. Relationship of mean $\mathrm{D}(-)-\beta$-hydroxybutyrate $(B H B)$ blood level to anticonvulsant effect in 18 patients maintained on the medium chain triglyceride diet for over 1 month.

4. The anticonvulsant effect appears most closely related to elevation in plasma levels of "ketone bodies" (primarily BHB and acetoacetate). Plasma levels of these substances are approximately equal during two different ketogenic regimens which have previously been shown to have comparable anticonvulsant effects $(6,7)$. A relationship between ketonemia and anticonvulsant effect is suggested by several other findings. It has long been known that the ketogenic diet has little anticonvulsant effect past early childhood. The present data indicate that this is associated with achievement of lower plasma ketone levels in the older child and adolescent. The mechanism by which susceptibility to ketonemia decreases with age is as yet obscure. Another relevant observation is that of gradually increasing anticonvulsant effect of the ketogenic diet over the first several days or weeks of diet therapy (1). Measurements of plasma BHB and acetoacetate levels in the present study indicate a concomitant gradual rise in plasma concentration of ketone bodies during the first month of therapy in children under the age of 10 years, the age group in which dietary therapy is likely to be effective. The most likely explanation is gradual depletion of glycogen stores, with progressively less availability of endogenous glucose. Finally, we have found a rapid loss of anticonvulsant effect after glucose is infused, with both worsening in the EEG and recurrence of seizure activity as plasma $\mathrm{BHB}$ and acetoacetate fall toward normal. These findings confirm earlier clinical observations on carbohydrate feeding by McQuarrie and Keith (8), and they add metabolic and electroencephalographic observations which have not been previously recorded during glucose administration to ketonemic children.

The rapid reversal of anticonvulsant effect after reduction in plasma BHB and acetoacetate suggests a direct anticonvulsant effect of these substances. However, the possibility that the anticonvulsant action of the ketogenic diet is mediated through ketonemia-induced secondary changes in cerebral metabolism is not excluded by the present findings. Ketonemia resulting from long term starvation has been found to produce profound changes in cerebral metabolism, with substantial substitution of BHB for glucose as the substrate for energy metabolism $(10,11)$. It has been suggested that this change may in some as yet unspecified manner reduce cerebral excitability $(1,6)$. This question, as well as the relationship between ketonemia and seizure threshold, is further defined in animal experiments which are the subject of a subsequent report.

\section{SUMMARY}

Metabolic effects of a ketogenic regimen in which ketonemia is induced by feeding of MCT are compared with those of the 


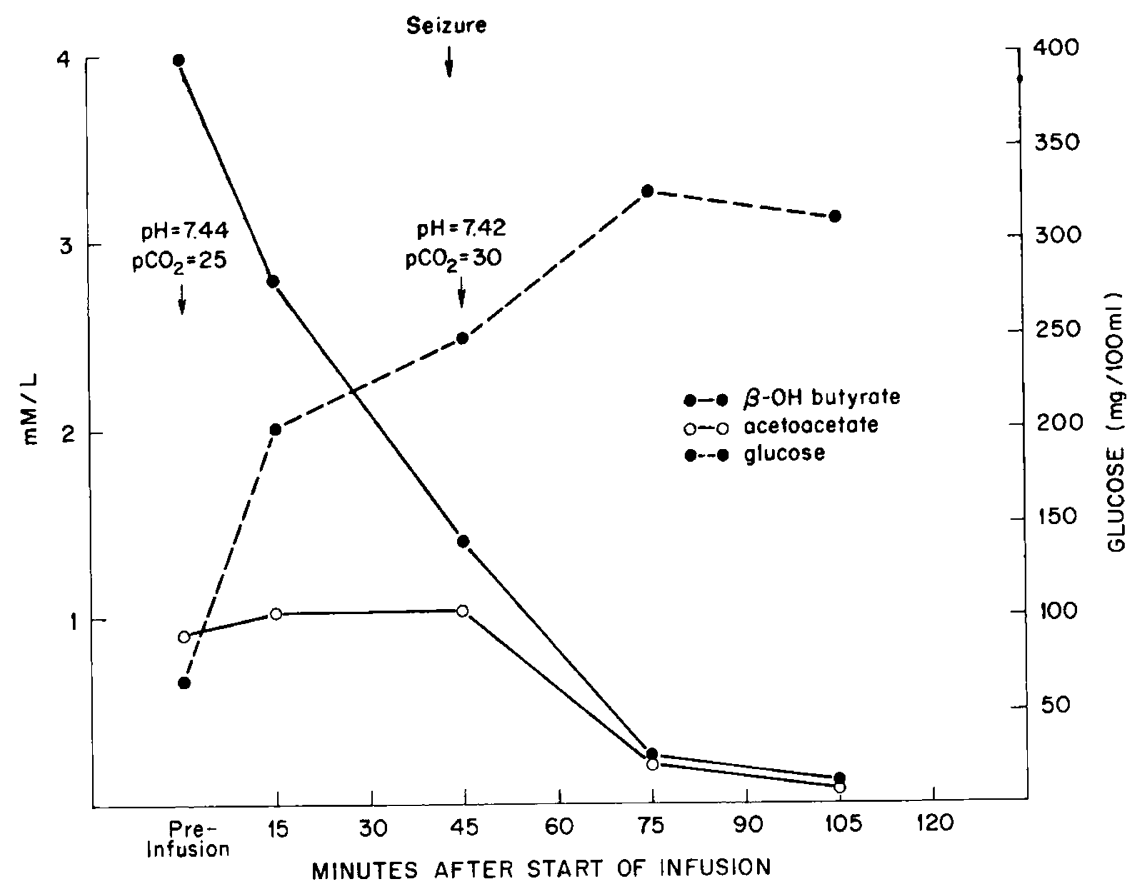

Fig. 2. Effect of glucose infusion in a 4-year-old boy with myoclonic seizures previously well controlled on the medium chain triglyceride diet. A single myoclonic seizure occurred after $45 \mathrm{~min}$, at which time plasma $\mathrm{D}(-)$ - $\beta$-hydroxybutyrate $(\beta-O H$ butyrate $)$ had fallen to about one-third of starting value. $\mathrm{pCO}_{2}$ increased slightly during this interval, but there was no significant change in venous $\mathrm{pH}$.

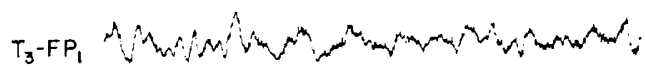

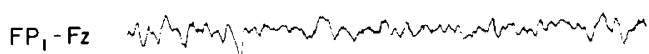

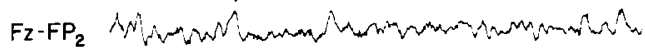

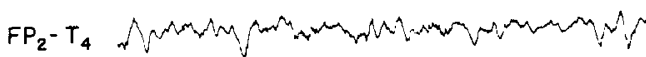

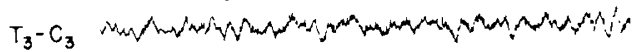

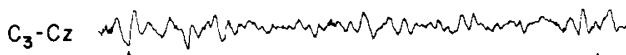

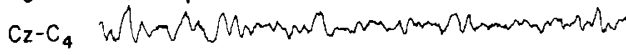

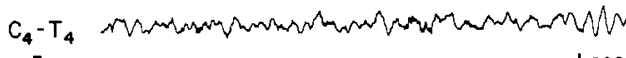

$$
\begin{aligned}
& \text { A }
\end{aligned}
$$

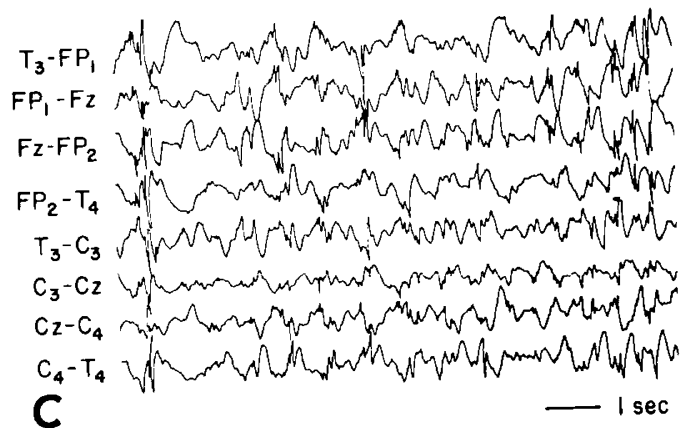

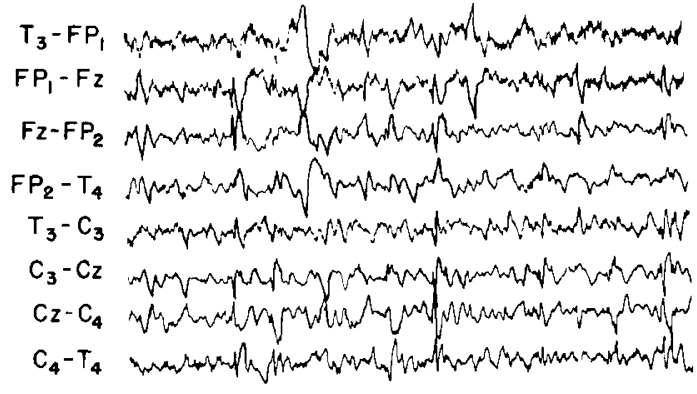

B

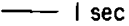

Fig. 3. Effect of glucose infusion on the EEG tracing of a 5-year-old boy with myoclonic seizures who had been well controlled on the medium chain triglyceride diet for over 1 year. $A$ : recording obtained immediately before glucose infusion. Background activity contains irregular slow and sharp waves, but there are no spike discharges. Plasma $D(-)-\beta$-hydroxybutyrate $=11.7 \mathrm{mM} . B: 30$ min after start of glucose infusion. There are increased sharp waves and occasional multifocal spike discharges. $C: 60 \mathrm{~min}$ after start of glucose infusion. Multifocal spike discharges now occur frequently and are of greater amplitude. $D: 90 \mathrm{~min}$ after start of glucose infusion. The high voltage polyspike and slow wave discharge pattern in the tracing was accompanied by a myoclonic seizure. Simultaneous plasma $\mathrm{D}(-)-\beta$-hydroxybutyrate $=4.3 \mathrm{mM}$.

standard high fat ketogenic diet. At approximately equal anticonvulsant potency these diets differ in their effects on plasma cholesterol and fatty acid levels, but produce similar elevations in BHB and acetoacetate. Seizure control in children maintained on ketogenic diets is positively correlated with plasma $\mathrm{BHB}$ levels.
Lowering of plasma $\mathrm{BHB}$ and acetoacetate by glucose infusion results in rapid loss of anticonvulsant effect. The results support the view that the anticonvulsant action of ketogenic diets is mediated by effects of BHB, acetoacetate, or both on cerebral excitability. 


\section{REFERENCES AND NOTES}

1. Appleton, D. B., and De Vivo, D. C.: An animal model for the ketogenic diet. Epilepsia, 15: 211 (1974)

2. Dekaban, A.: Plasma lipids in epileptic children treated with a high fat diet. Arch. Neurol., 15: 177 (1966).

3. De Vivo, D. C., Pagliara, A. S., and Prensky, A. L.: Ketotic hypoglycemia and the ketogenic diet. Neurology, 23: 640 (1973).

4. Hendley, D. D., Davenport, H. W., and Toman, J. E. P.: Effect of acid-base changes in experimental convulsive seizures. Amer. J. Physiol., 153: 580 (1948).

5. Huggett, A. St. G., and Nixon, D. A.: Use of glucose oxidase, peroxidase, and O-dianisidine in determination of blood and urinary glucose. Lancet, ii: 368 (1957).

6. Huttenlocher, P. R., Wilbourn, A. J., and Signore, J. M.: Medium-chain triglycerides as a therapy for intractable childhood epilepsy. Neurology, 2l. 1097 (1971).

7. Isom, J. B.: Treatment of minor motor seizures with MCT diet. Paper presented at the Annual Meeting of the Child Neurology Society, Madison, Wisconsin, October 12, 1974

8. McQuarrie, I., and Keith, H. M.: Epilepsy in children: Relationship of variation in the degree of ketonemia to occurrence of convulsions in epileptic children on ketogenic diets. Amer. J. Dis. Child, 34: 1013 (1927).

9. Millichap, J. C., Jones, J. D., and Rudis, B. P.: Mechanism of anticonvulsant action of ketogenic diet. Amer. J. Dis. Child., 107: 593 (1964).

10. Owen, O. E., Morgan, A. P., Kemp, H. G., et al.: Brain metabolism during fasting. J Clin Invest. 46: 1589 (1967).

11. Smith, A. L., Satterthwaite, H. S., and Sokoloff, L.: Induction of brain D(--)-beta-hydroxybutyrate dehydrogenase by fasting. Science, 163: 79 (1969).

12. Stern, I., and Shapiro, B.: A rapid and simple method for the determination of esterified fatty acids and for total fatty acids in blood. J. Clin. Pathol. 6: 15 (1953).

13. Talbot, F. B., Metcalf, K., and Moriarty, M.: Epilepsy: Chemical inestigation of rational treatment by production of ketosis. Amer. J. Dis. Child., 33: 218 (1927).

14. Uhlemann, E. R., and Neims, A. H.: Anticonvulsant properties of the ketogenic diet in mice. J. Pharmacol. Exp. Ther. 180: 231 (1972).

15. Wilder, R. M.: Effect of ketonuria on course of epilepsy. Mayo Clin. Bull., 2: 307 (1921).

16. Williamson, D. H., Mellanby, J., and Krebs, H. A.: Enzymic determination of $\mathrm{D}(-)$-beta-hydroxybutyrc acid and acetoacetic acid in blood. Biochem. J., 82: 90 (1962)

17. Zlatkis, A., Zak, B., and Boyle, A. J.: A new method for the direct determination of serum cholesterol. J. Lab. Clin. Med., 41: 486 (1953).

18. Sigma Chemical Co. St Louis, Mo.

19. Calibiochem, La Jolla, Calif.

20. Informed consent was obtained for all subjects in this study.

21. This research was supported by Grants nos. NS-09785 and F. R. 125, National Institutes of Health, Public Health Service.

22. Requests for reprints should be addressed to: P. R. Huttenlocher, Department of Pediatrics, University of Chicago, Chicago, Ill. 60637 (USA)

23. Accepted for publication December 3, 1975 .

\title{
Cholesterol Metabolism in Cultured Fibroblasts in Adrenoleukodystrophy
}

\author{
EPHRAIM YAVIN, AUBREY MILUNSKY,(30) G. ROBERT DeLONG, ANDREA H. NASH, AND
}

EDWIN H. KOLODNY

\begin{abstract}
Department of Biochemistry, The Eunice Kennedy Shriver Center, Walter E. Fernald State School, Waltham; Departments of Pediatrics and Neurology, Massachusetts General Hospital and Harvard Medical School, Boston,
\end{abstract} Massachusetts, USA

\section{Extract}

The basic biochemical defect of X-linked adrenoleukodystrophy (sudanophilic leukodystrophy, Schilder's disease) is unknown. To investigate reported abnormalities in cholesterol metabolism in vitro, we examined cultured skin fibroblasts of four patients and four normal control subjects. The kinetics of retention and accumulation of $\left[{ }^{14} \mathrm{C}\right]$ cholesterol by these cells was studied. After 3 days of exposure to tracer amounts of $\left.{ }^{14} \mathrm{C}\right]$ cholesterol, an apparent steady state between the medium and cellular cholesterol was established. The specific radioactivity expressed per $\mathrm{mg}$ of protein was similar for both Schilder and control fibroblasts. After labeling the pre-existing cellular cholesterol pool, the rate of loss of label was followed up for a 6-day period. About $23 \%$ and $14 \%$, respectively, of the cellular radioactivity in both Schilder's disease and control cells were released into the medium after the consecutive change with fresh nonlabeled medium. No significant differences in $\left[{ }^{14} \mathrm{C}\right.$ cholesterol rates of uptake or release were observed between control and Schilder's disease fibroblasts. About $44 \%$ of the labeled cholesterol was present in an esterfied form after incubation in the presence of unheated serum in both Schilder's and control cultures.

\section{Speculation}

These results do not confirm previously reported abnormalities in cholesterol metabolism in cultured fibroblasts. Nevertheless, accumulating evidence from studies of fatty acid composition of brain and adrenal tissues in Schilder's disease indicates the presence of an abnormal series of long chain fatty acids. Studies are now in progress to determine whether these possibly unique long chain fatty acids are evident in fibroblasts from patients with Schilder's disease.

The association of adrenoleukodystrophy (sudanophilic leukodystrophy, Schilder's disease, or diffuse cerebral sclerosis) and adrenal insufficiency has been recognized for over 50 years (17). This disorder has been clearly delineated as X-linked recessive and separated from the heterogenous group of fatal degenerative leukodystrophies characterized by other modes of inheritance $(6$, $7,15,18,19)$. Distinct heterogeneity exists in the X-linked form with variable severity of the adrenal cortical atrophy from asymptomatic to overt adrenal crises. The characteristic pathology includes widespread demyelination and gliosis of cerebral and 